\title{
Individual, health facility-related, and psychosocial determinants of retention in chronic HIV care among HIV-positive young people in Mukono Municipality, Uganda
}

\author{
Phillip Kasibante ${ }^{1,2}$, Jemimah Kyeyune Kiboss ${ }^{1}$, Christine Atuhairwe ${ }^{1}$, Ivan Mugisha Taremwa ${ }^{1}$ \\ ${ }^{1}$ Clarke International University, Kampala, Uganda \\ ${ }^{2}$ Ministry of Health, Kampala, Uganda
}

\begin{abstract}
Introduction: Retention in care is a known determinant of successful treatment outcome and serves as a measure of abating the emergence of resistant strains of human immunodeficiency virus (HIV). We report on the individual, health facility-related, and psychosocial determinants of retention in chronic HIV care among young HIV-infected patients in Mukono Municipality, in Uganda.

Material and methods: A cross sectional study included 118 participants, aged 15 to 24 years and health workers, and it was conducted between May and September 2017. Data were obtained using a questionnaire, an abstraction tool, and key informant interviews. Logistic regression analysis was used to establish predictor variables, with a $p \leq 0.05$.

Results: Slightly half, $53.4 \%$ of the HIV patients were retained in care for 24 months. Participants with CD4 cell counts above 500 cells/ $\mu \mathrm{l}$ at the initiation of treatment were 3 times more likely to be lost to follow-up (odd, $3: 1$ ). The availability of peer support services $(p=0.026)$, no extra support from parents/ friends $(p=0.02)$, stigma and associated blame on others $(p=0.04)$ showed statistical correlation to the retention in HIV chronic care. On the other hand, peer support services attendance $(p=0.091)$ and attitude of health providers $(p=0.762)$ did not show statistical significance in the retention of care of HIV patients.

Conclusions: The study reports a low retention in HIV care among people aged 15 to 24 years, and this was positively associated with individual, health facility-related, and psychosocial factors, which require intensive efforts to abate such barriers.
\end{abstract}

HIV AIDS Rev 2020; 19, 2: 99-105 DOI: https://doi.org/10.5114/hivar.2020.96386

Key words: retention, chronic care, HIV, young people, Mukono district.

\section{Introduction}

The Global AIDS Update 2017 reports the number of people living with human immunodeficiency virus (PLWHIV) to be 36.7 million [1]. East and Southern Africa have the high- est burden (19 million), of which 2.7 million of HIV-infected people are aged between 15 and 24 years $[1,2]$. In Uganda, there are 1.5 million people infected with HIV, with a prevalence of $6.0 \%$ [3]. Among people aged $15-24$ years, the HIV prevalence is $3.72 \%$ for women and $2.32 \%$ for men [4].

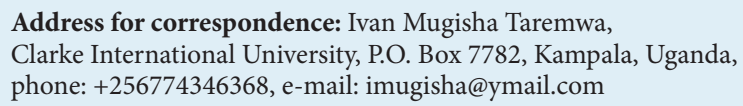

International Journal of HIV-Related Problems

HIV \& AIDS R e v i e w 
Mukono district has an HIV prevalence of $7.4 \%$, and it occurs more among young adults (15-19 years at $1.1 \%$ and $3.3 \%$ among those aged 20-24 years) [4]. Though not well studied, the high HIV burden among the 15-24 years group is associated with existing barriers relating to sexual education, limited knowledge on ways of preventing HIV transmission, and misconceptions [5].

As global efforts towards elimination of HIV infection intensifies, various mechanisms have advanced in diagnosis and care. The 90-90-90 strategy by UNAIDS predicts to have 90\% of HIV-positive persons diagnosed, of which $90 \%$ to be enrolled into anti-retroviral therapy (ART), of whom $90 \%$ to achieve virologic suppression by the year 2020 [6]. This paradigm requires strong and effectual ways premised on timely diagnosis, linkage and enrollment into care, ART initiation, retention in care, and adherence [6]. Despite the milestone in HIV diagnosis and intensification of ART in sub-Saharan Africa, various obstacles such as lost to follow-up and deaths remain high [7]. As HIV disease is incurable and requires long-term healthcare, the retention in constant care with ART is a key factor [8]. This starts when an HIV-infected person is linked to appropriate services, prepared for treatment, initiated with ART, and maintained in lifelong ART care [9].

The retention in HIV care is defined as continuous engagement of a patient from the time of diagnosis in a package of prevention, treatment, support, and care services. It begins from the moment of initial engagement in care, when a person with HIV is successfully connected to services, which assess for eligibility, initiation, and lifetime ART care [6]. This is a key determinant of successful HIV treatment, as it abates the emergence of drug resistant strains and viral suppression [10]. The average retention has been seen to decrease from $86 \%$ at 12 months to $82 \%$ at 24 months and $72 \%$ at 60 months [9], yet it is crucial in reducing morbidity and mortality. Thus, the study investigated individual, health facility-related, and psychosocial determinants of retention in chronic HIV care among HIV-positive young people in Mukono municipality, Uganda.

\section{Material and methods}

\section{Study design, site, and duration}

From May to September 2017, a cross-sectional study was conducted in Mukono municipality. Municipality is divided into two sub-counties, Goma and Central division. It is located in the periphery of the capital city and is one of the most populated districts. The study was carried out among four health facilities (Goma Health Centre III, Mukono Town Council HC IV, Mukono Church of Uganda HC IV, and Eseri Domiciliary Clinic). These facilities were purposively chosen as the main HIV service providers for people aged 15-24 years, as per the District Health Information System (DHIS2) report from 2017. The selection of these facilities was based on services reported in DHIS2 in the quarter of April-June 2017, health workers with lead- ership responsibility including facility in-charge, ART incharge, youth-friendly services with focal person and district HIV focal person, and leaders of young persons (peer leaders). The study employed both quantitative and qualitative methods of data collection.

\section{Sample size estimation}

This was obtained using Kish Leslie formula (1965) with $n=Z^{2} \mathrm{pq} / \mathrm{d}^{2}[11]$, where $n$ is the ideal sample size, $Z$ is the $95 \%$ confidence limit, $d$ is the maximum margin of error allowed, and $p$ is the estimated proportion of one-year retention among PLWHIV for adolescents and young people reported at $60 \%$ in Mukono district [12]. Therefore, we estimated a sample size of 133 participants.

\section{Selection criteria and sampling procedures}

The study included voluntary consented HIV-positive patients aged 15-24 years, who were able to hear and speak English and Luganda (a local language used in the area). Using probability to size proportion, the sample was computed based on the actual numbers enrolled at the facility, that is Goma Health Centre III (43 patients), Mukono Town Council HC IV (55 patients), Mukono Church of Uganda HC IV (55 patients), and Eseri Domiciliary Clinic (43 patients). Clinical registers of those centers with people aged 15-24 years living with HIV and receiving ART were used as the sampling frame. A sampling interval of 3 was used; therefore, every third person meeting the inclusion criteria was enrolled. On a clinic day, HIV patients were introduced to the research through a health talk conducted by a member of research team, and after this, all eligible participants were assented (those under the age of 18 years) and consented (participants aged 18 years and above). Participants' stratification by age was considered for 15-19 and 20-24 years. All qualified HIV patients who were scheduled and did not attend the clinic day were contacted by phone and through village health teams (VHTs). The participants were enrolled, and face to face interviews were conducted. Further, data was abstracted from participants' files to meet the required aspects. In addition, three key informants (KIs) from each selected ART facility were purposively enrolled, making a total of 12 KIs. Key informant interviews were conducted as face to face interviews and contents were analyzed for usefulness.

\section{Data collection and management}

Data were collected using an interviewer-administered questionnaire, key informant interview, and data abstraction tool. The questionnaire was availed in both English and Luganda after being pretested. The questionnaire and key informant interview were developed based on various studies previously conducted on this subject [6-10]. The topics 
covered in the questionnaire interview included personal aspects such as demographic features, medication, education level attained, psychosocial issues, and support as well as knowledge and their attitudes. Psychological aspects comprised of depression, drug/alcohol addiction, stigma, and discrimination. The health facility-related factors included counseling, medication availability, health workers attitudes, practices and adequacy, health worker knowledge and skills, youth friendly services availability and access, and social support services availability. The topics of key informant interviews focused on the policy availability and implementation, and patient's and psychological aspects of retention. The questionnaire and key informant interviews were administered by trained research assistants with a medical background, and the interview lasted for 25-40 minutes. The questionnaire was administered to HIV patients. The key informants included health workers (facility in-charge, ART in-charge, youth friendly services focal person), and district HIV focal person and leaders of young persons (peer leaders). These were selected based on their experience and role in ART care. The questions were adopted from previous measurements conducted by various researchers on this subject [6-10], and were administered by the principal investigator or a designee. The interview lasted for about $30 \mathrm{~min}$ utes, at the key informants' discretion. Data abstraction tools were used to collect retrospective data, with study variables included independent variables (individual, health facility, and psychological factors) and dependent variable being retention in chronic care among HIV-positive young people in Mukono municipality.

\section{Data management}

Data was entered using Epi data and analyzed with SPSS, version 18.0. The data was analyzed at univariate, bivariate, and multivariate levels. Variables of univariate and bivariate analyses with a $p$ value $\leq 0.05$ were considered statistically significant for multivariate analysis.

\section{Ethical considerations}

Ethical approval was obtained from the research and ethics committee of the Clarke International University. Additionally, the study obtained letters of authorization from Mukono municipality local council authorities. Participants offered informed assent/consent, and confidentiality was strongly upheld at all stages.

\section{Results}

\section{Analysis of individual factors}

\section{Analysis of retention in care}

The study approached 168 participants, of these, only 118 respondents were considered at the final stage with fully completed questionnaires. Among the study respondents, more than half (53.4\%) of the HIV chronic patients had been retained in care at 24 months.

\section{Socio demographic characteristics}

There were $31.4 \%$ male and $68.6 \%$ female. Among the participants, $33.1 \%$ were aged $15-19$ years and $66.9 \%$ were 20-24 years, as shown in Table 1.

\section{Individual factors}

There were $87.3 \%$ respondents who acknowledged availability of peer support services. Most respondents (95.8\%) had knowledge of how ART worked, and 98.3\% believed that ART improved their health. To lessen the side effects of ART, most participants were advised to consult a doctor if their condition deteriorated, ensure they rest more often, take a lot of fluids, green vegetables and fruits. However, there were chronic HIV patients that were discouraged from taking ART due to side effects such as nausea and jaundice. Also, the study participants reported challenges including sustaining their sources of income, as they spent a lot of time at the facility, rude health workers, balancing school work and counseling sessions, lack of transport, distant location of HIV care facilities, fear of their peers, and missing appointment days.

There were social barriers including shame, verbal abuse from relatives, feel of guilt, denied access to certain items, discrimination from fellow students or neighbors, having protected sexual intercourse at all the times, individual worry that one may not complete studies, inadequate finances, loss of appetite, low self-esteem, and self-imposed stigma.

On participants daily routines, most participants considered avoiding sex, consumption of a balanced diet, being stress-free, following the doctor's instructions, having their meals on time, moving with ART everywhere, eating lots of green vegetables and fruits, continuous encouragement and counseling, gaining spouse support, attending self-management classes, proper time management (especially regarding the medicines), keeping oneself busy or distracted to avoid negative thoughts, and having peer support to encourage positive living.

The individual factors analyzed indicated that gender $\left(24.0-38.1 \%\right.$ vs. $\left.39.0-61.9 \% ; \chi^{2}=2.852 ; p=0.091\right)$, age (25.0-39.7\% vs. $\left.38.0-60.3 \% ; \chi^{2}=2.686 ; p=0.101\right)$, mode of HIV transmission (12.0-19.0\% vs. $30.0-47.6 \% ; \chi^{2}=0.682$; $p=0.711)$, and the sort of people that lived with chronic HIV patients $\left(19.0-30.2 \% ; \chi^{2}=3.729 ; p=0.292\right)$ were not significantly associated with the retention of HIV care. On the other hand, the availability of peer support services was found to be significantly associated with retention of chronic HIV patients (59.0-93.7\% vs. $\left.4.0-6.3 \% ; \chi^{2}=4.931 ; p=0.026\right)$. Information from key informants revealed that males had poor health seeking behavior as given in this narrative: "I noticed that most males have poor health seeking behavior, so poor adherence to treatment and hence poor retention in care" (KI, Goma H/C III). 
Table 1. Demographic characteristics of respondents $(n=118)$

\begin{tabular}{|c|c|}
\hline Characteristics & Frequency (\%) \\
\hline \multicolumn{2}{|l|}{ Sex } \\
\hline Male & $37(31.4)$ \\
\hline Female & $81(68.6)$ \\
\hline \multicolumn{2}{|l|}{ Age } \\
\hline $15-19$ years & $39(33.1)$ \\
\hline $20-24$ years & $79(66.9)$ \\
\hline \multicolumn{2}{|l|}{ Mode of HIV transmission } \\
\hline Mother to child & $20(16.9)$ \\
\hline Horizontal & $60(50.8)$ \\
\hline Unknown & $38(32.2)$ \\
\hline \multicolumn{2}{|l|}{ Living with } \\
\hline Parents/guardian & $33(28.0)$ \\
\hline Relatives & $15(12.7)$ \\
\hline Partner & $38(32.2)$ \\
\hline Alone & $32(27.1)$ \\
\hline \multicolumn{2}{|l|}{ Orphan } \\
\hline Yes & $44(37.3)$ \\
\hline No & $74(62.7)$ \\
\hline \multicolumn{2}{|l|}{ Medication on ARVs } \\
\hline Yes & $117(99.2)$ \\
\hline No & $1(0.8)$ \\
\hline \multicolumn{2}{|l|}{ School issues in school } \\
\hline Yes & $43(36.4)$ \\
\hline No & $75(63.6)$ \\
\hline \multicolumn{2}{|c|}{ Availability of peer support services } \\
\hline Yes & $103(87.3)$ \\
\hline No & $15(12.7)$ \\
\hline \multicolumn{2}{|l|}{ Attends peer support services } \\
\hline Yes & $84(71.2)$ \\
\hline No & $34(28.8)$ \\
\hline \multicolumn{2}{|l|}{ Knowledge of how ARVs work } \\
\hline Yes & $113(95.8)$ \\
\hline No & $5(4.2)$ \\
\hline \multicolumn{2}{|c|}{ In the past one month, heard information on ARVs } \\
\hline Yes & $117(99.2)$ \\
\hline No & $1(0.8)$ \\
\hline \multicolumn{2}{|l|}{ Source of information } \\
\hline Schools/teachers & $12(10.2)$ \\
\hline Community & $10(8.5)$ \\
\hline Health facility & $16(13.6)$ \\
\hline Friends & $4(3.4)$ \\
\hline Magazines/newspapers & $9(7.6)$ \\
\hline TV health talk shows & $22(18.6)$ \\
\hline Internet & $2(1.7)$ \\
\hline Posters/billboards & $13(11.0)$ \\
\hline Radio & $26(22.0)$ \\
\hline
\end{tabular}

\section{Psychosocial factors}

Results indicated that young people had disclosed their HIV status to parents (40.7\%), relatives (26.3\%), friends (11.9\%), health workers (2.5\%), and others (18.6\%). At the time of disclosure, $49.2 \%$ did not react favorably, for instance, husbands chased away their wives and some were abandoned by their partners. The results showed that $21.2 \%$ had ART delivered to their homes by parents and relatives, while $18.6 \%$ of the respondents were escorted to the health facility by parents, relatives, or friends. Only $11.0 \%$ of the young people reported that they had received support in form of money, food, and transport from parents, husbands, or siblings. In the previous 3 months, the young people reportedly experienced shame (55.1\%), guilt (46.6\%), self-pity (48.3\%), blamed others (27.1\%), low self-esteem (36.4\%), and suicidal tendencies $(24.6 \%)$. Furthermore, participants reportedly did the following: chose not to attend social gatherings (11.0\%), isolated themselves from family and friends (12.7\%), decided not to get married $(16.1 \%)$, not to have sex $(28.0 \%)$, not to have more children (41.5\%), avoided going to the clinic (41.5\%), and to the hospital (7.6\%). Also, they reported their fearful events such as being gossiped about (55.9\%), being verbally insulted, harassed, and/or threatened (44.1\%), being physically harassed and/or threatened (39.0\%), and being physically assaulted (39.8\%). Some participants had been excluded from socio gatherings once or more often (14.4\% and $18.6 \%$, respectively). Also, $27.1 \%(n=32)$ of the participants drunk alcohol or used illicit drugs.

\section{Health system factors}

The key informants indicated that all participants knew about the standard reaction of young people in chronic HIV care, as stated in the narrative; "The policy is implemented by caring about young people living with HIV/ AIDS, regular follow ups, giving them ART after the client has tested positive for HIV and has been counseled. We also assay for their viral load every 6 months for those below 19 years" (KI, Eseri Clinic). Majority of HIV patients ( $n=89$, 75.4\%) lived in less than 5 kilometers from a health facility, and $53.4 \%$ of them spent 500 to 3,000 Uganda shillings on transport. Most (89.8\%) of the respondents acknowledged that young people were offered separate services, as highlighted in the narrative by a key informant: "The young people have their own counseling session and the location of HIV clinic" (KI, Mukono HC IV). Further, $83.1 \%$ of HIV services at a health facility were supported by the government, and therefore offered free of charge services. Majority (91.5\%) of chronic HIV patients had received counseling prior to the interview, and 95.8\% of the respondents found the healthcare workers friendly. Most $(81.4 \%)$ of the participants did not pay for health services. The level of adequacy of health services did not significantly influence the retention of young people in chronic HIV care in Mukono municipality $\left(16,25.4 \%\right.$ versus $28,44.4 \% ; \chi^{2}=3.524$; $p=0.474)$. Healthcare workers or services offered were not associated with the retention of young people in chronic HIV 
Table 2. Multivariate analysis and summary model

\begin{tabular}{|c|c|c|c|c|c|c|}
\hline Variable & $\begin{array}{l}\text { Coefficient } \\
\text { (B) }\end{array}$ & $\begin{array}{c}\text { Standard error } \\
\text { (SE) }\end{array}$ & Wald $\chi^{2}$ & Df & Sig. $(p>z)$ & $\begin{array}{l}\text { Odds ratio exp } \\
\text { (B) }\end{array}$ \\
\hline \multicolumn{7}{|l|}{ Availability of peer support services } \\
\hline Yes & -0.978 & 0.771 & 1.612 & 1 & 0.204 & 0.376 \\
\hline No" & 0.000 & & & & & 1.000 \\
\hline \multicolumn{7}{|l|}{ Received extra support } \\
\hline Yes & 1.763 & 0.729 & 5.846 & 1 & 0.016 & 5.828 \\
\hline No" & 0.000 & & & & & 1.000 \\
\hline Blaming others for one's status & -0.879 & 0.471 & 3.485 & 1 & 0.062 & 0.415 \\
\hline \multicolumn{7}{|l|}{ Sex } \\
\hline Male & -0.292 & 0.457 & 0.408 & 1 & 0.523 & 0.747 \\
\hline Female\# & 0.000 & & & & & 1.000 \\
\hline \multicolumn{7}{|l|}{ Age } \\
\hline $15-19$ years & -0.759 & 0.472 & 2.579 & 1 & 0.108 & 0.468 \\
\hline $20-24$ years $^{\#}$ & 0.000 & & & & & 1.000 \\
\hline \multicolumn{7}{|l|}{ Having peer support } \\
\hline Yes & -0.301 & 0.541 & 0.310 & 1 & 0.578 & 0.740 \\
\hline $\mathrm{No}^{\#}$ & 0.000 & & & & & 1.000 \\
\hline Constant & 1.578 & 1.894 & 0.694 & 1 & 0.405 & 4.845 \\
\hline
\end{tabular}

-2Log likelihood 143.939; Cox and Snell $R^{2} 0.149$; Nagelkerke $R^{2} 0.200$

\#Reference category

care $\left(15,23.8 \%\right.$ vs. $\left.36,57.1 \% ; \chi^{2}=4.505 ; p=0.342\right)$; thus, it did not translate into the maintenance of their clientele level, as presented in Table 2 .

The healthcare providers in a key informant interview listed the following challenges in retaining young people living with HIV in chronic care: adherence, pregnancy among females, limited resources, and lost to follow-up due to stigma.

\section{Discussion}

In this study, more than half (53.4\%) of HIV chronic patients had been retained in care at 24 months. This indicates a poor retention rate and is much below the target of 90-90-90 strategy, where $90 \%$ of HIV cases ought to be retained in chronic care. To this, efforts are vital to optimize the retention in HIV care and reduce the related morbidity, mortality, incidence of new infections, and development of ART resistance. This result compares to the target of the Ministry of Health under the AIDS Control Program, ensuring that at least $95 \%$ of HIV patients are retained in chronic care by strengthening comprehensive patient appointment and tracking systems, implementing evidence-based communication strategy, client counseling, and education services such as adherence and psychosocial support [13].

In this study, gender, age, mode of HIV transmission, attendance of peer support services, and kind of people that lived with the HIV patients did not influence their retention in care. Only availability of peer support services was a sig- nificant predictor of retention in ART care. The retention in chronic HIV care among people aged 15-24 years presents unique challenges. This is attributed to the fact that this sub-population is often dependent upon a caregiver, which makes individual characteristics of the caregiver a paramount determinant. While some studies have indicated that age is significantly associated with loss from engagement in care [14-16], this was not the case in our study. This difference is attributed to varied settings, sample size, and sociodemographic variables among study populations. With regards to gender, findings of this study did not show statistical significance to the retention in care. This contradicts previous studies $[14,16]$. There were more females retained in care as compared to males, a finding that has been already established [1, 16-18]. This is partly explained by the fact that males tend to be involved in various activities, thus such services ought to be extended to such places - a phenomenon that requires directed strategies. In this study, health facility factors such as distance covered by HIV patients, money spent, separate youth services, and the attitude of health workers towards patients did not influence their retention in care $(p>0.05)$. This is contrary to previous findings [19-23]. While it is a wide phenomenon, it looks like frequent appointments translate to high transportation costs and absence at work. Further, home responsibilities and drug stock-out contribute significantly to low ART retention. Health service delivery factors such as lack of agespecific and comprehensive care package for adolescents and young adults has also been reported to significantly influ- 
ence the retention of this sub-population in ART care [24]. Recent strategies adopted by the Ministry of Health to 'test and treat' as per the WHO recommended guidelines has a significant impact on the long-term retention care since persons are initiated early enough, thus reducing early mortality presently seen during the first year following positive testing.

In our study, a psychosocial factor 'blame on others' was found to significantly influence the retention of young people in chronic HIV care. However, in multivariate analysis, this was not significant. This is attributed to fear of disclosure and rationalization [25]. Therefore, strategies targeting non-disclosure including couple counseling can help in retaining patients in ART care. Lack of extra support from parents/ friends was found to significantly influence the retention in HIV care. Other studies have indicated that retention in care is highly related to stigma, discrimination, and social exclusion $[26,27]$. This encourages traditional healers or home treatment of self-diagnosed illnesses versus conventional medical interventions.

Our study has established the individual, health facilityrelated, and psychosocial determinants of retention in chronic HIV care among young people. Whereas the study findings are depictive of the studied population, the results ought to be interpreted with a caution due to limited sample size.

\section{Conclusions}

This study reports a low retention in HIV care among young people in Mukono municipality. This is attributed to perceived/actual stigma, inadequate support from parents, peers, and friends, health workers attitudes, and feeling of 'being' healthy at initiation in chronic care. Based on our study, a substantial improvement in readiness to counsel before enrollment, reduction in stigma, support from parents, friends, and peers should be considered as key markers of retention in care among HIV patients aged 15 to 24 years. Furthermore, early identification of HIV-infected young people coupled with strong linkages and active tracking should be implemented to ensure the retention of all HIV-positive young people in the test-and-treat era.

\section{Ethics approval and consent to participate}

We received ethical approval from research and ethics committee of the Clarke International University (formerly known as the International Health Sciences University). All respondents provided written informed consent/assent after receiving detailed description of the study. Eligible participants were assented/consented in privacy and confidentiality was ensured. As we did not have any minor below the age of 16, we did not consider seeking consent from their guardians/parents.

\section{Acknowledgements}

We are grateful to all study participants, key informants, and village health teams who participated in our work.

\section{Conflict of interest}

The authors declare no conflict of interest with respect to the research, authorship, and/or publication of this article.

\section{References}

1. Global AIDS Update. Available at: https://www.scribd.com/document/354266907/Global-AIDS-Update; 2017.

2. WHO/UNAIDS/UNICEF. Towards Universal Access. Scaling up priority HIV/AIDS interventions in the health sector. Progress report, 2010.

3. UNAIDS Progress report: Global HIV/AIDS response. Epidemic update and health sector progress towards universal access. Available at: http://whqlibdoc.who.int/publications/2011/9789241502986_ eng.pdf. 2011

4. Uganda Population-Based HIV Impact Assessment UPHIA; 2017.

5. The Uganda HIV and AIDS Country Progress Report; 2016.

6. WHO. Retention in HIV programs; defining the challenges and identifying solutions, meeting report. Geneva: WHO; 2011.

7. Fox MP, Rosen S. Patient retention in antiretroviral therapy programs up to three years on treatment in sub-Saharan Africa: systematic review. Trop Med Int Health 2010; 15 Suppl 1: 1-15.

8. National Antiretroviral Treatment and Care Guidelines for Adults, Adolescents and Children. Available at: http://www.who.int/hiv/amds/ uganda_moh_treatment_guidelines.pdf (Accessed: 14.03.2017).

9. Ronoh JC, Mwaniki P, Mutai JK, Njenga S, Mutisya AK. Factors associated with retention in care among HIV positive adults attending Pumwani Comprehensive Care Centre, in Nairobi County, Kenya; 2017.

10. Emma SK, Scott B, Michael JM. The HIV treatment cascade and care continuum: updates, goals, and recommendations for the future. AIDS Res Ther 2016; 13: 35

11. Leslie K. Survey Sampling. $1^{\text {st }}$ ed. New York: John Wiley and Sons; $1965,45-47$.

12. Uganda Bureau of Statistics. The National Population and Housing Census 2014 - Area Specific Profile Series, Kampala, Uganda; 2017.

13. Ministry of Health. Consolidated guidelines for prevention and treatment of HIV in Uganda; 2017.

14. Nakigozi G, Makumbi FE, Kigozi G, et al. Barriers to utilization of HIV care services among adolescents and young adults in Rakai, Uganda: the role of economic strengthening. Global Social Welfare 2015; 2: 105-110.

15. Bergmann H, Pitorak H, Cornman H. Linkage and retention in preART care: best practices and experiences from fourteen countries. Arlington: USAID's AIDS Support and Technical Assistance Resources, AIDSTAR-One, Task Order 1; 2013.

16. Lahuerta M, Lima J, Nuwagaba-Biribonwoha H, et al. Factors associated with late antiretroviral therapy initiation among adults in Mozambique. PLoS One 2012; 7: e37125.

17. Kuznetsova AV, Meylakhs AY, Amirkhanian YA, et al. Barriers and facilitators of HIV care engagement: results of a qualitative study in St. Petersburg, Russia. AIDS Behav 2016; 20: 2433-2443.

18. Hoffmann M, MacCarthy S, Batson A, et al. Barriers along the care cascade of HIV infected men in a large urban center of Brazil. AIDS Care 2016; 28: 57-62.

19. Buregyeya E, Kulane A, Colebunders R, et al. Tuberculosis knowledge, attitudes and health-seeking behaviour in rural Uganda. Int J Tuberc Lung Dis 2011; 15: 938-942.

20. Geng EH, Glidden DV, Bwana MB, et al. Retention in care and connection to care among HIV-infected patients on antiretroviral therapy in Africa: estimation via a sampling-based approach. PLoS One 2011; 6: e21797.

21. WHO. Antiretroviral therapy for HIV infection in adults and adolescents: recommendations for a public health approach; 2010. 
22. Cheever LW. Engaging HIV-infected patients in care: their lives depend on it. Clin Infect Dis 2007; 44: 1500-1502.

23. Mahy M, Tassie JM, Ghys PD, et al. Estimation of anti-retroviral therapy coverage: methodology and trends. Curr Opin HIV AIDS 2010; 5: 97-102.

24. Mark D, Armstrong A, Andrade C, et al. HIV treatment and care services for adolescents: a situational analysis of 218 facilities in 23 sub-Saharan African countries. J Int AIDS Soc 2017; 20 (Suppl 3): 21591.

25. Darshine G, Nathan F, Katharina K. Risk factors barriers and facilitators for linkage to antiretroviral therapy care; a systematic review AIDS 2012; 26: 2059-2067.

26. Galarraga J. Hispanic-American Culture and Health. Available at: http://www.cwru.edu/med/epidbio/mphp439/ Hispanic_Healthcare.pdf. 2012.

27. Morrison K. Stigma, discrimination, and HIV prevention among MARPS and OVPs: implications for the Caribbean. Caribbean Regional HIV Prevention Summit on Most-at-Risk Populations; 2011. 